\title{
I-year follow-up of a randomized controlled trial comparing seclusion and mechanical restraint in people with serious mental illness
}

\author{
Jan Bergk*, Michael Birk and Tilman Steinert
}

Address: ZfP Weissenau, Dep. of Psychiatry I, University of Ulm, Weingartshoferstr. 2, 88214 Ravensburg, Germany

* Corresponding author

from WPA Thematic Conference. Coercive Treatment in Psychiatry: A Comprehensive Review

Dresden, Germany. 6-8 June 2007

Published: 19 December 2007

BMC Psychiatry 2007, 7(Supp| I):PI8 doi:I0.II86/I47I-244X-7-SI-PI8

This abstract is available from: http://www.biomedcentral.com/I47I-244X/7/SI/PI8

(c) 2007 Bergk et al; licensee BioMed Central Ltd.

\section{Background}

Seclusion and mechanical restraint are widely used for people with serious mental disorders. In most countries one intervention is preferred while the other is considered as inhuman or not sufficiently safe, but identical arguments refer to different preferences. There is a lack of evidence from well-designed studies on compulsory measures in psychiatry.

\section{Methods}

We conducted a cohort study with optional randomisation comparing seclusion and mechanical restraint among in-patients with acute psychotic disorders. We determined an ethical aspect as main outcome variable: the restriction of human rights from the patients' point of view, measured by a scale developed for this purpose, Human DIgnity during COercive Procedures, DICOP. In addition we screened for posttraumatic stress disorder (PTSD). After one year a follow-up was conducted by telephone interviews. We reassessed the incidence of PTSD after coercive measures by the Impact of Event Scale (IES).

\section{Results}

102 out of 233 patients exposed to coercive measures within 24 months could be included, 26 could be randomized (12 seclusion, 14 restraint). 60 patients could be contacted for follow-up interviews. Further results will be presented. 